\title{
An Introduction to water quality analysis
}

Roy, Ritabrata

Hydro-Informatics Engineering, under Department of Civil Engineering, National Institute of Technology Agartala, Barjala, Jirania, Tripura, INDIA

Corresponding Author: ritroy@gmail.com

\section{A R T I C L E I N F O}

Received: 07 July 2018 | Accepted: 17 October 2018 | Published Online: 31 December 2018

DOI: $10.31786 / 09756272.18 .9 .2 .214$

EOI: 10.11208/essence.18.9.2.214

Article is an Open Access Publication.

This work is licensed under Attribution-Non Commercial 4.0 International

(https://creativecommons.org/licenses/by/4.0/)

CThe Authors (2018). Publishing Rights @ MANU_ICMANU \& ESSENCE-IJERC.

\section{A B S T R A C T}

Water is perhaps the most precious natural resource after air. Though the surface of the earth is mostly consists of water, only a small part of it is usable, which makes this resource limited. This precious and limited resource, therefore, must be used with care. As water is required for different purposes, the suitability of it must be checked before use. Also, sources of water must be monitored regularly to determine whether they are in sound health or not. Poor condition of water bodies are not only the indictor of environmental degradation, it is also a threat to the ecosystem. In industries, improper quality of water may cause hazards and severe economic loss. Thus, the quality of water is very important in both environmental and economic aspects. Thus, water quality analysis is essential for using it in any purpose. After years of research, water quality analysis is now consists of some standard protocols. There are guidelines for sampling, preservation and analysis of the samples. Here the standard chain of action is discussed briefly so that it may be useful to the analysts and researchers.

\section{K E Y W O R D S}

Water Quality Assessment | Water Quality Analysis | Chain of Custody

\section{I T A T I O N}

Roy, Ritabrata (2019): An Introduction to water quality analysis. ESSENCE Int. J. Env. Rehab. Conserv. IX (1): $94-100$. 


\section{Introduction}

\section{What is Water Quality?}

Water Quality can be defined as the chemical, physical and biological characteristics of water, usually in respect to its suitability for a designated use.

Water can be used for recreation, drinking, fisheries, agriculture or industry. Each of these designated uses has different defined chemical, physical and biological standards necessary to support that use. For example, there are stringent standards for water to be used for drinking or swimming compared to that used in agriculture or industry.

\section{What is Water Quality Analysis?}

After many years of research, water quality standards are put in place to ensure the suitability of efficient use of water for a designated purpose. Water quality analysis is to measure the required parameters of water, following standard methods, to check whether they are in accordance with the standard.

\section{Why Water Quality Analysis is required?}

Water quality analysis is required mainly for monitoring purpose. Some importance of such assessment includes:

1. To check whether the water quality is in compliance with the standards, and hence, suitable or not for the designated use.

2. To monitor the efficiency of a system, working for water quality maintenance

3 . To check whether upgradation / change of an existing system is required and to decide what changes should take place.

4. To monitor whether water quality is in compliance with rules and regulations.

Water quality analysis is of extremely necessary in the sectors of:

1. Public Health (especially for drinking water)

2. Industrial Use.

\section{Procedures of Water Quality Analysis}

The general flow of procedures for water quality analysis is mentioned in Chart-1

Chart-1: General Flow of Procedures for Water Quality Analysis

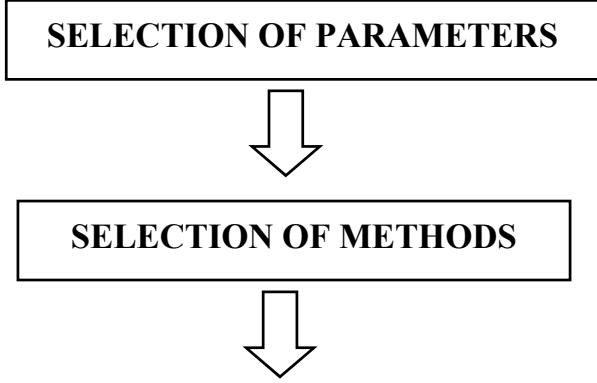

PRECISION AND ACCURACY OF METHOD SELECTED AS PER REQUIREMENT

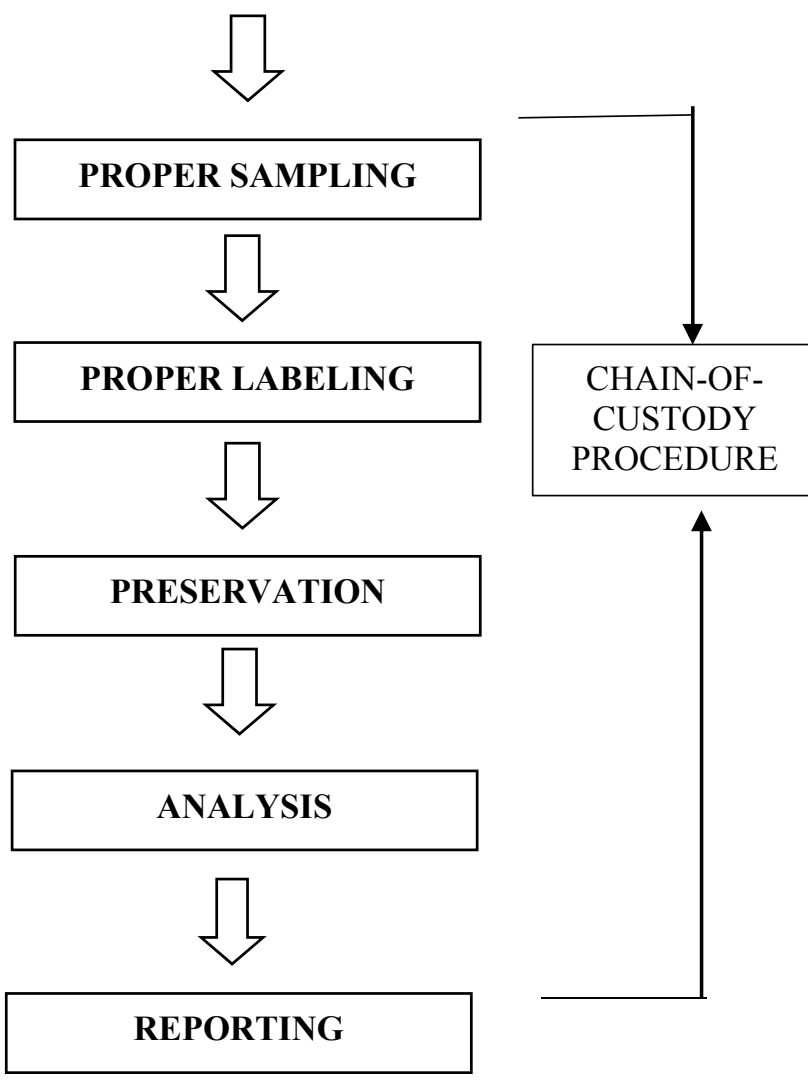

\section{Selection of Parameters}

The parameters of water quality are selected entirely according to the need for a specific use of that water. Some examples are:

Drinking: As per WHO/CPCB Standards 


\section{Irrigation:}

$\mathrm{pH}$

Conductivity

Sodium \& Potassium

Nutrients

Specific compounds
Industries: As per specific requirement

Domestic Consumption: As per BIS Standards

Water Bodies: As per CPCB guidelines

However, some of the most common parameters followed for checking potability and industrial use in India are in Chart-2

\section{Chart-2: Parameters for Water Quality Analysis}

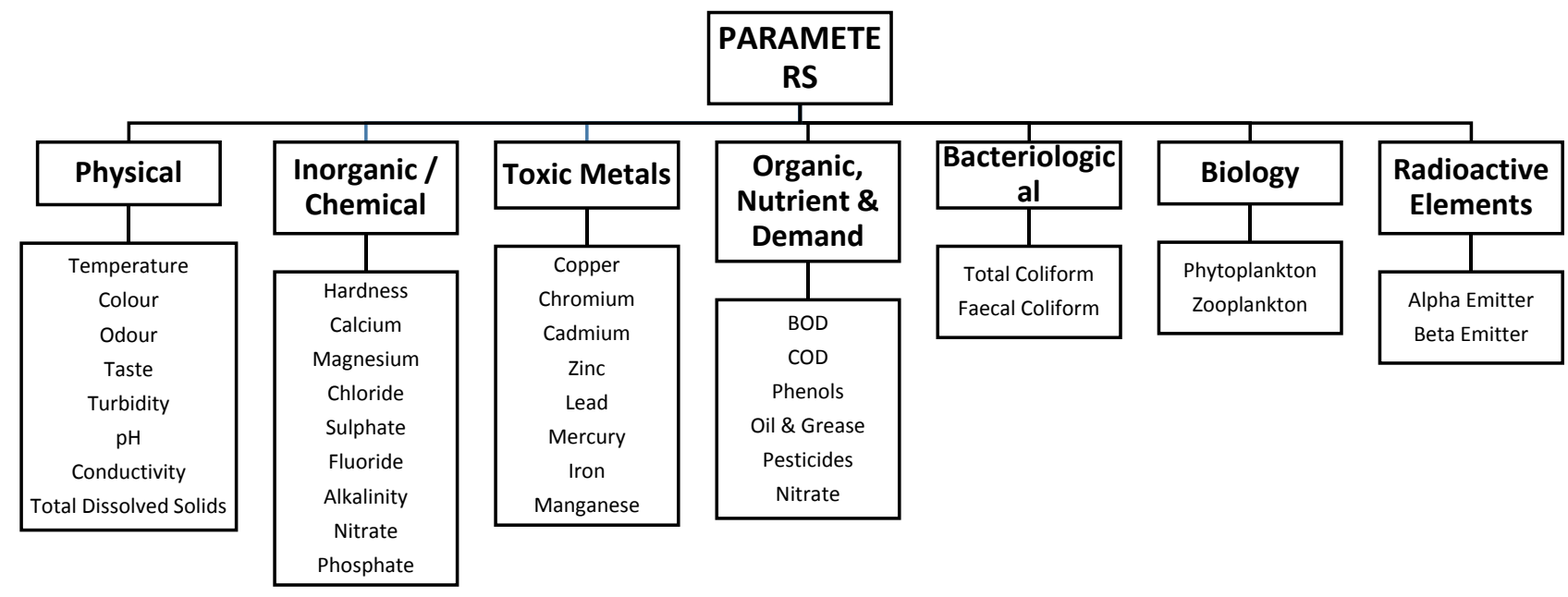

\section{Selection of Methods}

The methods of water quality analysis are selected according to the requirement. The factors playing key role for the selection of methods are:

(i) Volume and number of sample to be analysed

(ii) Cost of analysis

(iii) Precision required

(iv) Promptness of the analysis as required

\section{Precision and Accuracy of Method Selected as Per Requirement}

What precision and accuracy to be maintained against a particular method is selected according to the need. The factors influencing this decision includes:
(i) Cost
(ii) Parameter
(iii) Use

\section{Chain-of-Custody Procedures}

Properly designed and executed chain-of-custody forms will ensure sample integrity from collection to data reporting. This includes the ability to trace possession and handling of the sample from the time of collection through analysis and final disposition. This process is referred to as "chainof-custody" and is required to demonstrate sample control when the data are to be used for regulation or litigation. Where litigation is not involved, chain-of-custody procedures are useful for routine control of samples.

A sample is considered to be under a person's custody, if it is in the individual's physical possession, in the individual's sight, secured and tamper-proofed by that individual, or secured in an area restricted to authorized personnel. The following procedures summarize the major aspects of chain-of-custody: 
(i) Sample labels: Labels are used to prevent sample misidentification as well as to identify the collector, if required. In other words, labelling ensures the responsibility and accountability of the collector.

(ii) Sample seals: Sample seals are used to detect unauthorized tampering with samples up to the time of analysis. So, it is essential to seal a sample before leaving the custody of the collector. Sealing must be done in such a way as one have to break the seal to access the sample.

(iii) Field logbook: Record all information pertinent to a field survey or sampling in a bound $\log$ Book is a good practice. At least the following data should be in the logbook:

(a) Purpose of sampling

(b) Location of sampling point

(c) Name and address of field contact

(d) Producer of material being sampled and address, if different from location

(e) Type of sample

(f) Method, date, and time of preservation.

(iv) Sample analysis request sheet: The sample analysis request sheet accompanies samples to the laboratory. The collector completes the field portion of such a form that includes most of the pertinent information noted in the log book. The laboratory portion of such a form is to be completed by laboratory personnel and includes: name of person receiving the sample, laboratory sample number, date of sample receipt, condition of each sample (i.e., if it is cold or warm, whether the container is full or not, color, if more than one phase is present, etc.) and determinations to be performed.

(v) Sample delivery to the laboratory: Sample(s) should be delivered to laboratory as soon as practicable after collection, typically within 2 days. Where shorter sample holding times are required, special arrangements must be made to insure timely delivery to the laboratory.
Where samples are shipped by a commercial carrier, the waybill number to be included in the sample custody documentation. Samples must be accompanied by a complete chain-of-custody record and a sample analysis request sheet.

(vi) Receipt and logging of sample: In the laboratory, the sample custodian inspects the condition and seal of the sample and reconciles label information and seal against the chain-ofcustody record before the sample is accepted for analysis. After acceptance, the custodian assigns a laboratory number, logs sample in the laboratory logbook and/or computerized laboratory information management system, and stores it in a secured storage room or cabinet or refrigerator at the specified temperature until it is assigned to an analyst.

(vii) Assignment of sample for analysis: The laboratory supervisor usually assigns the sample for analysis. Once the sample is in the laboratory, the supervisor or analyst is responsible for its care and custody.

(viii) Disposal: Samples are held for the prescribed amount and duration for the project or until the data have been reviewed and accepted. Samples are disposed usually after documentation. However, disposal must be in accordance with local, state, and U.S. EPA approved methods.

\section{Proper Sampling}

Proper sampling is a vital condition for correct measurement of water quality parameters. Even if advanced techniques and sophisticated tools are used, the parameters can give an incorrect image of the actual scenario due to improper sampling. The proper sampling should fulfil the following criteria:

1. Representative: The data must represent the wastewater or environment being sampled. So, the 
following factors must be well planned for proper sampling:

(i) Process of Sampling

(ii) Sampling size/volume

(iii) Number of Sampling Locations

(iv) Number of Samples

(v) Type of Samples

(vi) Time Intervals

During sampling, these factors must also be taken care of:

(a) Choosing of proper sampling container

(b) Avoiding contamination

(c) Ensure the personal safety of the collector

2. Reproducible: The data obtained must be reproducible by others following the same sampling and analytical protocols.

3. Defensible: Documentation must be available to validate the sampling procedures. The data must have a known degree of accuracy and precision.

4. Useful: The data can be used to meet the objectives of the monitoring plan.

Proper Labeling

Proper labelling prevents sample misidentification and ensures the responsibility and accountability of the collector. The sample container should be labelled properly, preferably by attaching an appropriately inscribed tag or label. Alternatively, the bottle can be labelled directly with a water-proof marker. Barcode labels are also available nowadays.

Information on the sample container or the tag should include at least:

(i) Sample code number (identifying location)

(ii) Date and time of sampling

(iii) Source and type of sample

(iv) Pre-treatment or preservation carried out on the sample

(v) Any special notes for the analyst (vi) Sampler's name

\section{Preservation}

There is usually a delay between the collection and analysis of a sample. The nature of the sample can be changed during this period. Therefore proper preservation is required in the way to laboratory after collection, and in the laboratory upto when analysis starts.

Complete and unequivocal preservation of samples, whether domestic wastewater, industrial wastes, or natural waters, is a practical impossibility because complete stability for every constituent never can be achieved. At best, preservation techniques only retard chemical (especially, hydrolysis of constituents) and biological changes that inevitably continue after sample collection.

No single method of preservation is entirely satisfactory; the preservative is chosen with due regard to the determinations to be made. Preservation methods are limited to $\mathrm{pH}$ control, chemical addition, the use of amber and opaque bottles, refrigeration, filtration, and freezing.

\section{Analysis}

The samples, after reaching laboratory, are analyzed, according to the requisite parameters, following standard methods and protocols.

\section{Reporting}

The ultimate procedure of water analysis is to prepare a proper repost against the submitted requisition. The repost must be authenticated before handing over the authority, submitted the samples for testing. All date should be kept in the laboratory $\log$ and preferably in laboratory database.

An alternative way to present the overall quality of water is to express it in the form of Water Quality Index (WQI). WQI is a concise numerical representation of overall water quality of a water 
body, which is convenient to interpret and used widely. WQI expresses the overall quality of water with a single digit, instead of many digits for all the WQP. Thus, it is readily conceivable for common people.

\section{Conclusion}

Assessment of water quality is essential to check the suitability of a water source for the designated use. Several water quality parameters are assessed and compared with their standard values to determine the acceptability of the source of water. After prolonged research, the procedures for the assessment of the water have also been standardized for different purposes. In this article such guidelines are discussed concisely in one place for the convenience of the researchers and analysts. Thus, it may be helpful for them to get an overview of the water quality assessment standards and procedures.

\section{References}

APHA and AWWA (1999): Standard Methods for the Examination of Water and Wastewater. American Public Health Association (APHA), 20th Ed, Washington, D.C., USA.

Brown, R. M.; McClelland, N. I.; Deininger, R. A. and Tozer, R. G. (1970): A water quality index- do we dare? Water and Sewage Works, October, 339-343.

BIS (2012): Indian Standard Drinking Water Specification (Second Revision).

Deshpande, L.; undated Water Quality Analysis: Laboratory Methods. National Environmental Engineering Research Institute (NEERI), Nagpur, Council of Scientific \& Industrial Research, New Delhi, Govt. of India

Kori, R.; Parashar, S. and Basu, D. D.; Undated Guide Manual: Water and Wastewater
Analysis. Central Pollution Control Board, Ministry of Environment and Forest, India.

Metcalf, E. and Eddy, H. (2003): Wastewater Engineering: Treatment and Reuse. Tata McGraw-Hill Publishing Co Ltd, India.

Roy, R. (2018): An Approach to Develop an Alternative Water Quality Index Using FLDM. In: Majumder M. (eds) Application of Geographical Information Systems and Soft Computation Techniques in Water and Water Based Renewable Energy Problems. Water Resources Development and Management. Springer, Singapore, 5168.

Roy, R. and Majumder, M. (2017): Comparison of surface water quality to land use: a case study from Tripura, India. Desalination and Water Treatment, 85, 147-153.

Roy, R. and Majumder, M. (2018): A Quick Prediction of Hardness from Water Quality Parameters by Artificial Neural Network. International Journal of Environment and Sustainable Development, 17(2/3): 247-257.

Matta, G. (2014): "A study on physico-chemical Characteristics to assess the pollution status of river Ganga in Uttarakhand." Journal of Chemical and Pharmaceutical Sciences, 7(3): 210-217.

Matta, G. and Kumar, A. (2015): Monitoring and Evaluation of River Ganga System in Himalayan Region with Reference to Limnological Aspects. World Applied Sciences Journal, 33 (2): 203-212.

Matta, G. and Kumar, A. (2017): Health Risk, Water Hygiene, Science and Communication. ESSENCE Int. J. Env. Conser. Rehab, 8(1): $179-186$. 
Matta, G. and Laura, G. (2016): Mercury, lead and arsenic: impact on environment and human health India. Journal of Chemical and Pharmaceutical Sciences, 9(2): 718 725 .

Matta, G.; Bhadauriya, G. and Singh, V. (2011): Biodiversity and Sustainable Development: A Review. ESSENCE - Int J for Env Rehab and Conser, 2(1): $72-$ 80.

Matta, G.; Laura, G.; Kumar, A. and Machel, J. (2018): Hydrochemical characteristics and planktonic composition assessment of River Henwal in Himalayan Region of Uttarakhand using CPI, Simpson's and Shannon-Weaver Index. Journal of Chemical and Pharmaceutical Sciences, 11(1).

Tyagi, S.; Sharma, B.; Singh, P. and Dobhal, R. (2013): Water Quality Assessment in Terms of Water Quality Index. American Journal of Water Resources, 1(3): 34-38.

WHO (2004): Guidelines for Drinking-Water Quality (3rd edition). 